\title{
A Two-Step Model Reduction Method for a Strap- on Launch Vehicle
}

\author{
Liu Pan, Guo Shao-Jing and Cai Guo-Ping \\ Department of Engineering Mechanics, State Key Laboratory of Ocean Engineering, Shanghai Jiaotong Univer- \\ sity, Shanghai 200240, P. R. China
}

\begin{abstract}
(Received 22 April 2013; revised: 15 September 2013; accepted 5 February 2014)
In this paper, a two-step model reduction method is proposed using a strap-on launch vehicle as research object. In this method, the double-compatible free-interface modal synthesis method is first used for the modeling of the system, and the first step of reduction work is done by preserving the lower-order modes of flexible components of the system in the modeling process for the system. Then the second step of reduction is done for the established dynamic model of the system using the modal cost analysis method. After the two steps of order reduction, a low-order dynamic model of the system can be obtained. Simulation results indicate that this low-order model can reflect the characteristics of the original system effectively and the order is low enough as well.
\end{abstract}

\section{INTRODUCTION}

It is well known that flexible components are used increasingly in aerospace structures due to limits on the weight of the launch vehicle. Moreover, to deliver more payloads to outer space, there is a trend to fit launch vehicles with long thrusters in order to carry more fuel; this results in the obvious flexibility of the launch vehicle. The introduction of flexibility makes dynamic modelling and the control of the system much more difficult. To describe the dynamic behaviour of the system exactly, flexible characteristics in the system should be adequately taken into account during the modelling process. This results in a high order of the established dynamic model. However, control design and implementation require that the order of the system should be as low as possible. Therefore, model reduction work should be done in order to obtain a low-order model convenient for control design. This low-order model should be able to reflect the dynamic characteristics of the original system, and its order should also be low enough. On the other hand, from the point of dynamic simulation, model order should not be high so as to improve computational efficiency.

For linear structure, lower-order modes play a main role in the dynamic response of the structure. So the lower-order modes are often preserved to form a reduction model to represent the original system approximately. This is the so-called modal truncation technique which has been widely applied in both theoretical research and engineering application. Essentially, the modal synthesis technique is a method used to build a low-order dynamic model for a complex system using the modal truncation technique. In using the modal synthesis method, the complex system is divided into several components reasonably, and the high-order dynamic equation of each component is first established using the finite element method (FEM). Then, the modal truncation technique is applied to the FEM equation in order to obtain a low-order modal dynamic equation of the component. Finally, the dynamic equation of the system is established by doing the modal synthesis for all the components based on the displacement and force compatibility conditions at the interfaces of the components. The first step of the modal reduction of the two step-method proposed in this paper is the local reduction of the system. This is when the order reduction in the modal synthesis method is done for the components in the modelling process for the system. Up to now, some different types of modal synthesis methods have been proposed by researchers according to the different selections of the modal functions of flexible components. ${ }^{1-4}$ The typical ones are the free-interface modal synthesis method and the fixed-interface modal synthesis method. Wang et al. modified the free-interface modal synthesis method and proposed the double-compatible free-interface modal synthesis method, a method that can achieve higher computation precision than the free-interface and fixed-interface modal synthesis methods. $^{4,5}$ The modal synthesis method is applicable to the dynamic modelling of large and complex engineering structures, and its validity has been justified in many practical applications. ${ }^{6-11}$ For modern large-scale aerospace structures, the modal synthesis method is still an important and effective analysis tool. For example, a space station is mainly composed of several cabins that are sent into space at different times. Generally, a ground test needs to be done for every cabin before sending it into space. However, the overall ground test of a space station is usually impossible. In this case, the modal synthesis method is available to get the characteristics of the space station by synthesizing the data of every cabin.

Other than the modal synthesis method mentioned above, there is another kind of model reduction technique in which the reduction is done after the system model is established. We call this technique the integral reduction of the system, which is the second step of the two-step model reduction method discussed in this paper. The integral reduction technique can be mainly divided into two classes: the dynamic condensation technique and the criteria reduction technique. These two techniques have both been studied deeply by researchers and widely applied in practical engineering. ${ }^{12,13}$ Furthermore, the dynamic condensation technique has been implemented in some commercial dynamics analysis software such as Nastran, Ansys, etc. For a spacecraft, the criteria reduction method is often used. The commonly used criteria include the inertia completeness criteria, the modal cost analysis criteria, and the balanced reduction criteria. ${ }^{14-19}$ Among these criteria, the modal cost analysis criteria has been extensively studied since it is 